\title{
EL SELLO "HECHO EN PERÚ" EN LA DECISIÓN DE COMPRA DE DETERGENTES Y CONSERVAS DE PESCADO POR LOS CONSUMIDORES DEL CERCADO DE AREQUIPA, 2012
}

\author{
THE "MADE IN PERU” STAMP IN THE PURCHASE \\ DECISION OF DETERGENTS OR TUNA FISH CANS \\ BY COSTUMERS IN AREQUIPA
}

Ximena Dongo Gárate y Michelle Segales Valdivia Universidad Católica San Pablo, Arequipa, Perú

\section{Resumen:}

En este artículo se exponen los resultados de un estudio descriptivo que valora la decisión de compra de detergentes y conservas de pescado por los consumidores y además el grado de etnocentrismo al consumo de los productos nacionales por medio de un cuestionario aplicado del 11 al 23 de noviembre del 2012. Se trabajó con una muestra de 259 mujeres con una edad mínima de 18 años de edad del Cercado de Arequipa, a quienes se les aplicó el cuestionario junto a unas tarjetas de ayuda visuales. Se encontró que el origen del producto no es un factor determinante a la hora de comprar los productos analizados. Sin embargo, los participantes indicaron su preferencia de compra por los productos nacionales a la hora de precisar su etnocentrismo contrariamente a lo expresado en la primera parte del cuestionario. Por último, la mayoría de los participantes conocían el sello "Hecho en Perú" especialmente por campañas televisivas y demostraban su apego a dicho sello, sin embargo no sabían quienes promovían la iniciativa.

Palabras Clave: Sello "Hecho en Perú", etnocentrismo al consumo, decisión de compra, país de origen. 


\begin{abstract}
:
In this paper, we expose the results of a descriptive study that values the purchase decision of detergents and fish conserves for the consumers and in addition the degree of ethnocentrism to the consumption of the national products by means of a questionnaire realized from November 11th to November 23th 2012. We worked with a sample of 259 women with an average age of 18 to more years of age of Arequipa's Center to whom the questionnaire was applied with cards of visual help. We found that the origin of the product is not a determinant factor at the moment of buying the analyzed products. Nevertheless, the participants indicated their preference of purchase to the national products at the moment of his ethnocentrism needed contrary to the expressed in the first part of the questionnaire. Finally, the majorities of the participants knew the stamp "Made in Peru" especially for television campaigns and were demonstrating his attachment to the above mentioned stamp, however there did not know those who were promoting the initiative.
\end{abstract}

Key words: Stamp "Made in Peru ", ethnocentrism to the consumption, decision of purchase, country origin.

\section{Introducción}

Son muchos los libros en los que se ha hablado de la importancia del proceso de compra, de hecho es uno de los temas empresariales más comentados ya que dicho proceso es el producto de factores externos y personales que influyen en el momento en que las personas deciden qué producto comprar. Es por eso que las empresas idean estrategias para hacer más atractivo su producto de manera que tengan características que los diferencien del resto de los productos. Si bien es cierto, en un principio se creía que el precio era el factor decisivo en el proceso de compra se ha demostrado que no es así, el proceso de decisión de compra es mucho más complejo. De acuerdo a Francisco López (2008) al tomar los economistas una racionalidad estricta y universal han exagerado la importancia del factor económico, obviando la influencia decisiva de otros factores como el psicológico, sociológico, cultural, etc., en dicha conducta. Para entender el comportamiento del consumidor, se debe conocer que los consumidores realizan un proceso a la hora de elegir un producto que muchas veces realiza de manera inconsciente. De acuerdo a Ferrell y Hartline (2006), existe un proceso de decisión de compra el cual consta de cinco etapas: en un primer momento el consumidor reconoce una necesidad, luego pasa por las etapas de búsqueda de información, evalúa todas las alternativas posibles, decide qué producto adquirir y realiza una evaluación después de la compra, verificando si el producto satisfizo sus expectativas. No obstante, este proceso no es igual en todas las decisiones 
de compra ya que existen factores tanto en el individuo como el ambiente que predisponen que se realice la compra. Es por ello que es necesario entender la conducta del comprador en cada etapa y las influencias que están operando: las actitudes de otros, los factores de situación inesperados y el riesgo percibido podrían afectar la decisión de comprar, etc.

La globalización está forzando a las empresas a operar y comportarse según ciertos estándares y expectativas. Esta tendencia se refleja inevitablemente en la forma en que las empresas comercializan sus productos internacionalmente (Sosa y Ortiz, s/f). El desarrollo de estrategias de marketing efectivas, que sean sensitivas a las diferencias culturales a través de los países, es de considerable importancia para el éxito en el mercadeo global (Gurhan-Canli \& Maheswaran, 200o). Precisamente, Jiménez y Calderón (2004) sostienen que ante la existencia de la diversidad de productos en el mercado, las empresas buscan posicionarse respecto a sus competidores a través de las características distintivas del producto. Así se buscan desarrollar características que atraigan la atención de los consumidores ya sea por innovación, diseño, distribución, etc. Sin embargo, no siempre es necesario crear una característica distintiva, existen factores del proceso productivo que ayudan a identificar a un producto de otro. Uno de ellos es precisamente el país de origen. Las actitudes de los consumidores hacia los productos de una nación en particular son sumamente importantes para lograr dicho éxito (Nagashima, 1970), y miden las fortalezas y las debilidades competitivas de una nación en el mercado internacional
(Agbonifoh \& Elimimian, 1999). El concepto del país de origen es una pista de información crítica sobre el producto, el cual juega un rol principal en que el producto sea aceptado en diferentes mercados del mundo (Gestiopolis, 2010). Justamente, la Asociación Americana del Marketing (1960) define el término país de origen o country of origin (COO en sus siglas en inglés) de los productos como el efecto que el país de origen de un producto tiene sobre las percepciones de calidad de un producto. El estudio de este efecto se enmarca dentro del área de comportamiento del consumidor, en la medida que el país de origen se identifica como una señal extrínseca que los consumidores emplean para hacer inferencias de los productos y los atributos de los mismos (Montesinos \& Curras, s/f).

El hecho de estudiar el efecto país de origen de manera aislada o junto con otros atributos ha dado lugar a dos perspectivas distintas de investigación (Lampert \& Jaffe, 1998). Por una parte, el enfoque de atributo único que analiza cómo afecta a los consumidores el conocer el país de origen como un factor más entre otros estudios incorporan el país de origen como un factor más entre otros como la marca, la calidad, el precio o el riesgo percibido, tratándose de un enfoque multiatributo. El enfoque multiatributo es el más aceptado ya que describe la situación de una forma más próxima a la realidad donde los consumidores evalúan el producto y sus atributos extrínsecos e intrínsecos dentro de un contexto global de compra ya sea por la categoría de producto, conocimiento de la marca o del fabricante, conocimiento y creencia del país de fabricación, etc. Montesinos y Currás (s/f) afirman que el efecto 
país de origen no es uniforme en todas las categorías de producto, ni en todos los lugares se manifiesta del mismo modo, sino que este efecto viene moderado por una serie de variables que provocan que éste tenga mayor o menor intensidad. Así el país de origen queda moderado por la familiaridad y el conocimiento del producto, la marca del producto y el uso de su información, por el grado de implicación con la categoría de producto o servicio y la experiencia que se tenga con la misma, así como el etnocentrismo del consumidor y sus características sociodemográficas.

El país de origen es una forma de diferenciar el producto de los competidores. Schooler (1965) se considera generalmente como el primer investigador en estudiar empíricamente este efecto, descubriendo que los productos, idénticos en todos los aspectos excepto en su país de origen, se perciben de manera diferente por los consumidores. Desde entonces, cientos de estudios se han publicado sobre este tema (Usunier, 2006). La investigación de Schooler muestra que el país de origen tiene un impacto en las percepciones de la calidad de los consumidores de un producto, así como en última instancia, la preferencia y disposición a comprar dicho producto. Además, varios estudios han demostrado que los consumidores tienden a tener una preferencia en relación a los productos de su propio país, o pueden tener una preferencia relativa por o aversión a los productos que vienen de ciertos países, los llamados afinidades y animosidades (Shimp \& Sharma, 1987). Así se puede afirmar que los consumidores evalúan los productos basados en el país donde fueron producidos y, que en general, los consumidores privilegian las alternativas producidas nacionalmente (Schnettler et al., 2008). Respecto a lo último, existe un término que define el hecho que los consumidores prefieran los productos nacionales que los extranjeros llamado "el etnocentrismo en el consumo" El etnocentrismo (Schnettler et al, 2010) es un factor importante para predecir las actitudes y percepciones de los consumidores hacia los productos extranjeros o importados ya que influencia los hábitos de compra del consumidor generando lealtad hacia su país y rechazo hacia otros países, de modo que la preferencia por productos nacionales se asocia a un alto grado de etnocentrismo en el consumo.

Muchas han sido las investigaciones que han querido determinar el efecto del "Origen de País" dentro de la preferencia de compra. Por ejemplo Berta Schnettler y sus colaboradores (2010) investigaron el efecto del origen del producto en la población chilena a la hora de elegir un producto de primera necesidad, en este caso el azúcar, y determinaron que en Los Ángeles y Temuco (Chile), en términos generales, el atributo país de origen fue más importante en relación al envase y al precio en la elección de azúcar producida en Chile e importada. Además se distinguieron dos tipologías de consumidores para quienes la relevancia al país de origen en la compra del azúcar es diferente, pero independientemente de la importancia asignada al origen, la totalidad de los consumidores chilenos encuestados mostró preferencia hacia el producto chileno; esto evidenció la importancia del efecto país de origen en la compra de este producto básico de alimentación en el sur de Chile. 
En Colombia el país de origen no es significativo a la hora de la compra de acuerdo a una investigación realizada por Concha et al. (2011) en el cual se evaluaban el efecto del país de origen en cervezas al concluir que para el caso colombiano los consumidores son personas seguras y racionales a la hora de tomar la decisión de comprar un producto de consumo masivo, como lo es en este caso la cerveza, que tienen en cuenta los atributos del producto y los beneficios que este ofrece en lugar de la procedencia de la cerveza, es decir, que para los colombianos el país de origen no es importante a la hora de comprar.

Mientras que en España se investigó dicha influencia en producto hortícolas (Guerrero et al., 2010) la cual mostró que los españoles si bien consideraban el origen del país como algo importante, el factor más decisivo era la frescura del producto. Ya que la investigación puso en manifiesto que la relevancia del país de origen dentro del proceso de evaluación del consumidor, si bien no se trata del que mayor importancia tiene ya que la frescura del producto adquiere mayor protagonismo debido principalmente a que la frescura es el atributo clave para la evaluación de calidad en productos alimenticios, por encima de aspectos como la calidad, precio o la reputación de la marca $\mathrm{u}$ origen. Adicionalmente, los resultados obtenidos ponen de manifiesto cierta tendencia etnocéntrica en la evaluación del origen de los productos hortícolas. El conocimiento de esta característica del comportamiento fue fundamental, dado que los consumidores españoles muy etnocéntricos tienden a procesar la información sobre las marcas extranjeras a un nivel muy inferior, por lo que están predispuestos de antemano a juzgar las marcas nacionales muy favorablemente en comparación a las foráneas, sin que el nivel de información sobre éstas sea quizás necesario (Supphellen y Rittenburg, 2001). Por el contrario, los consumidores españoles menos etnocéntricos procesaron la información relativa a las marcas extranjeras de un modo diferente, estando dispuesto a conocer todos los aspectos relativos a las mismas, de modo que la evaluación de dichas marcas será más objetiva y la formación de una opinión estará más fundamentada. Igualmente, estos consumidores suelen acumular información acerca de los atributos y características de los productos extranjeros mientras que los consumidores muy etnocéntricos españoles simplemente desarrollan estructuras cognitivas con un contenido que incluye más estereotipos que información real (Supphellen y Rittenburg, 2003).

En el caso peruano, tanto el Estado como el sector privado lanzaron una propuesta para incentivar a la población a consumir productos peruanos a través de la campaña “Cómprale al Perú". Dicha campaña nació en el 2004 dirigida a generar conciencia en la ciudadanía nacional a comprar y consumir productos producidos en el Perú. De manera de garantizar no sólo el incremento del empleo sino también el crecimiento de la economía (El Comercio, 2009). La iniciativa de la campaña también era promovida por diversas empresas del medio, lo cual se plasmó en una reunión de gremios empresariales y de emprendedores, el nacimiento oficial de crear el eslogan "Cómprale al Perú”. Al mismo tiempo en medio de la reunión del Acuerdo Nacional, en la mesa de Promoción del 
Empleo, los representantes políticos allí reunidos, contemplaron también esta idea. Se conversó sobre la necesidad de orientar el incremento de las capacidades de producción de las fábricas peruanas. Eso significaba buscarles mercado tanto externo como interno nacional (Ministerio de la Producción, 2006).

Con el fin de identificar aquellas empresas unidas a la campaña surgió la necesidad de crear un sello distintivo que permita a los consumidores conocer aquellos productos que cuentan con la certificación de calidad que promueve el Estado. Así nació el sello "Hecho en Perú", el sello al igual que la campaña busca promover la calidad y la venta de los productos elaborados en el Perú, como un medio concreto para contribuir con la generación de riqueza y de empleo en nuestro país (El Peruano, 2011). Dicho programa tiene como prioridad promover la calidad de los productos elaborados en el Perú, por considerar ésta la vía de promoción más segura y sostenible de una oferta productiva competitiva a nivel nacional e internacional. Así en la página de INDECOPI (2009) dice lo siguiente: "Cada vez la decisión de compra de los consumidores no sólo depende de la intención de adquirir productor peruanos, sino sobre todo adquirir productos de calidad a precios competitivos" (párr. 19).

Es por ello, dentro del Programa el Sello de Calidad "Hecho en Perú (Made in Peru)" tiene un rol protagónico, ya que no sólo identifica si el producto ha sido elaborado en el Perú, sino a su vez, certifica que dichos productos cumplan con estándares de calidad requeridos por el mercado. El programa busca mejorar la competitividad de los productos elaborados en el país, sin discriminar el origen del capital de las empresas productoras, y a su vez sensibiliza al consumidor peruano y extranjero a que ante iguales condiciones en la oferta, decida orientar su compra por productos peruanos que tengan el sello de calidad "Hecho en Perú (Made in Peru)".

En tal sentido, es de gran interés conocer cómo actúan los compradores locales ante la exposición y promoción de los productos bandera del Perú, ya que la mayoría de los consumidores peruanos se ven en la posición de elegir entre productos nacionales e importados. Por lo tanto es de gran importancia determinar si el Sello "Hecho en Perú" está teniendo el efecto deseado de manera que se logre conocer si la población se siente identificada y prefiere comprar los productos que lleven dicha marca. Al no haber trabajos anteriores, la investigación se justifica por el valor que tiene el tema del consumo de productos nacionales por parte de los consumidores peruanos en el territorio nacional relacionado con la influencia de la difusión de "Hecho en Perú" en el país, precisamente en la ciudad de Arequipa. Por tanto nuestro estudio responde a la siguiente pregunta de investigación: ¿Cuál es el impacto del sello "Hecho en Perú" en la decisión de compra de productos nacionales por los consumidores del Cercado de Arequipa?

\section{Método}

\section{Muestra}

La muestra está conformada por mujeres de 18 a más años de edad y pertenecientes a la PEA (Población Económicamente Activa) de la Ciudad de Arequipa (Cercado), ya que ellas son las que toman la decisión final de comprar uno u otro producto, además de 
contar con poder adquisitivo para realizar estas compras ya sea para el consumo en el hogar como individual. Además de tener los suficientes conocimientos acerca de la realidad y entorno de nuestro país, es decir, tienen mayor conocimiento del tema.

La cantidad de participantes se determinó mediante el muestro probabilístico de corte sistemático simple con un nivel de confianza de $95 \%$, de una población de 10,839.58 mujeres (PEA, Cercado), obteniendo de esta manera una muestra de 249 mujeres, con 10 participantes adicionales por cuestiones de la mortalidad muestral. Adicionalmente, se utilizó la técnica de muestreo por estratos, para categorizar a las unidades de análisis en función de la edad en el cual las mujeres empiezan a contar con mayor poder adquisitivo, ya que se encuentran formando un hogar o con edad suficiente para vivir solas. Para ello, se tomaron los valores de la muestra y la población, obteniendo así, la constante $\mathrm{fh}=\mathrm{n} / \mathrm{N}$, el cual nos da un resultado de fh $=0.024$ (ver Tabla 1$)$.

\section{Tabla 1.}

Distribución de la muestra por edades

\begin{tabular}{|c|c|c|}
\hline $\begin{array}{c}\text { Grupos } \\
\text { de edades }\end{array}$ & $\begin{array}{c}\text { Población } \\
\text { Cercado }\end{array}$ & Muestra \\
\hline 18 a 29 años & 3794 & 90 \\
\hline 30 a 39 años & 2927 & 70 \\
\hline 40 a 49 años & 2276 & 55 \\
\hline 50 a 59 años & 1301 & 31 \\
\hline 60 a más años & 542 & 13 \\
\hline Total & 10,840 & 259 \\
\hline
\end{tabular}

\section{Instrumentos}

Como instrumentos de investigación se utilizaron un cuestionario y unas cartillas de apoyo visual. Los cuales permitieron la recolección de datos precisos de los participantes de la muestra. Mediante esta técnica se pudo medir la actitud de los consumidores respecto al sello "Hecho en Perú" difundida en el Cercado de la ciudad.

El cuestionario fue elaborado por las autoras al no existir un instrumento adecuado para medir las variables del conocimiento y la preferencia de compra de productos con el sello "Hecho en Perú". Este cuestionario contiene 16 preguntas, las cuales se dividieron en la pregunta filtro de edad y dos apartados. El primer apartado constó de 8 preguntas cerradas acerca del conocimiento de marcas, preferencia de compra del producto, características que le hacen preferir dicha marca y que datos en el empaque del producto lee antes de la compra, si es que leyera dicha información; todo esto en el caso de conservas de pescado y detergentes. En cuanto al segundo apartado, constó de 8 preguntas las cuales analizaban el nivel de etnocentrismo de los participantes en cuanto a la preferencia de compra de productos nacionales y el conocimiento acerca del sello "Hecho en Perú", sus promotores y el sentimiento del participante acerca de esta campaña nacional.

Por otro lado, las cartillas visuales utilizadas fueron fotografías de las cinco marcas de conservas de pescado y detergentes, eligiendo las marcas más conocidas en el mercado. Asimismo, las encuestas aplicadas fueron totalmente anónimas, de aplicación auto dirigida. Además de contar con las cartillas de ayuda con las imágenes de los productos a investigar, las cuales estuvieron impresas en papel bond A-5, a color con fondo blanco. Se utilizó este instrumento 
ya que se pudo aplicar en poco tiempo y nos permitió recabar información precisa de los consumidores arequipeños.

\section{Procedimientos}

La recolección de datos se realizó en el lapso de dos semanas, del 11 al 23 de noviembre del 2012. Los participantes fueron encuestados en los exteriores de los supermercados y mercados del centro de la ciudad de Arequipa, donde las autoras solicitaron con amabilidad la participación de las personas y se les informó sobre el tema y motivo de la encuesta, así como también el anonimato de la misma para obtener una mayor veracidad.

Los lugares elegidos fueron: los supermercados "El Súper", "Franco" y "Plaza Vea” (La Marina) y el mercado "San Camilo". Durante la realización de la misma, las autoras estuvieron junto al encuestado en todo momento para disipar cualquier duda que se pudiera presentar y al término de la encuesta se agradeció por su participación. Los datos obtenidos se procesaron en hojas de cálculo de Excel 2007 y en el programa SPSS 15.

\section{Resultados}

Para el procesamiento de los datos se ha utilizado estadísticos descriptivos. La muestra de mujeres encuestadas está dividida en cinco grupos de edades obtenidas por el método de muestreo probabilístico estratificado. Teniendo el mayor porcentaje (34\%) de encuestadas entre el rango de 18 a 29 años de edad, ya que en la población del Cercado viven más mujeres de esa edad.
Tabla 2.

Porcentajes de la muestra por edades

\begin{tabular}{|c|c|c|}
\hline Edad & Cantidad (fi) & Porcentaje (pi\%) \\
\hline $18-29$ & 88 & $34 \%$ \\
\hline $30-39$ & 71 & $27 \%$ \\
\hline $40-49$ & 56 & $22 \%$ \\
\hline $50-59$ & 32 & $12 \%$ \\
\hline $60-$ más & 12 & $5 \%$ \\
\hline Total & 259 & $100 \%$ \\
\hline
\end{tabular}

Analizando el primer apartado del cuestionario, el cual está conformado por las preguntas del número 1 al 8 , se tiene que el $100 \%$ de las participantes conocen las marcas mostradas en ambos productos analizados (conservas de pescado y detergentes). En cuanto a conservas de pescado el 29\% de las participantes prefieren comprar "Campomar", siendo esta la marca con mayor preferencia por encima de "Fanny" (22\%), "Atún Real” (20\%),"Florida” (15\%) y “Bayovar" (14\%).

Tabla 3.

Porcentajes de preferencia de marcas de conservas

\begin{tabular}{|l|c|c|}
\hline $\begin{array}{l}\text { Pregunta 2: } \\
\text { ¿Cuál prefiere } \\
\text { usted comprar? }\end{array}$ & $\begin{array}{c}\text { Cantidad } \\
\text { (fi) }\end{array}$ & $\begin{array}{c}\text { Porcentaje } \\
\text { (pi\%) }\end{array}$ \\
\hline Bayovar & 37 & $14 \%$ \\
\hline Atún real & 51 & $20 \%$ \\
\hline Campomar & 76 & $29 \%$ \\
\hline Fanny & 56 & $22 \%$ \\
\hline Florida & 39 & $15 \%$ \\
\hline Total & 259 & $100 \%$ \\
\hline
\end{tabular}

La decisión de adquirir dichos productos se basa principalmente por tener variedad en sus presentaciones y por ser producto nacional, con un $24 \%$ cada una, mientras que el precio con $14 \%$ no resulta ser un factor determinante (ver Tabla 4). 
Tabla 4.

Porcentajes de decisión de compra de conservas

\begin{tabular}{|l|c|c|}
\hline $\begin{array}{l}\text { Pregunta 3: } \\
\text { ¿En qué basa } \\
\text { su decisión de } \\
\text { adquirirlo? }\end{array}$ & Cantidad (fi) & Porcentaje (pi\%) \\
\hline $\begin{array}{l}\text { La marca es } \\
\text { conocida }\end{array}$ & 47 & $18 \%$ \\
\hline $\begin{array}{l}\text { Por su calidad } \\
\text { reconocida }\end{array}$ & 52 & $20 \%$ \\
\hline Por ser nacional & 62 & $24 \%$ \\
\hline Por su precio & 37 & $14 \%$ \\
\hline $\begin{array}{l}\text { Por sus } \\
\text { presentaciones }\end{array}$ & 259 & $24 \%$ \\
\hline Total & & $100 \%$ \\
\hline
\end{tabular}

En cuanto a detergentes el 28\% de los participantes prefieren comprar "Ariel”; sin embargo "Sapolio" es la segunda marca más comprada con un $26 \%$, a pesar de contar con un menor porcentaje, la diferencia es mínima, por lo que, se puede decir que ambas marcas son las preferidas por las consumidoras arequipeñas; estando esas por encima de "Bolívar" (19\%), "Ace" (14\%) y "Noritt" (12\%).

\section{Tabla 5.}

\section{Porcentajes de preferencia de marcas de detergentes}

\begin{tabular}{|l|c|c|}
\hline $\begin{array}{l}\text { Pregunta 5: ¿Cuál } \\
\text { prefiere usted } \\
\text { comprar? }\end{array}$ & Cantidad (fi) & $\begin{array}{c}\text { Porcentaje } \\
\text { (pi\%) }\end{array}$ \\
\hline Ace & 37 & $14 \%$ \\
\hline Ariel & 73 & $28 \%$ \\
\hline Bolívar & 50 & $19 \%$ \\
\hline Sapolio & 67 & $26 \%$ \\
\hline Noritt & 32 & $12 \%$ \\
\hline Total & 259 & $100 \%$ \\
\hline
\end{tabular}

La decisión de adquirir dichos productos (detergentes) se basa en dos factores, primero por la marca con $27 \%$ y luego por ser un producto nacional con $26 \%$, mientras que el precio no resulta ser un factor tan determinante en la elección del producto, ya que cuenta con el menor porcentaje $12 \%$ (ver Tabla 6).

Tabla 6.

\section{Porcentajes de decisión} de compra de detergentes

\begin{tabular}{|l|c|c|}
\hline $\begin{array}{l}\text { Pregunta 6: } \\
\text { ¿En que basa } \\
\text { su decisión de } \\
\text { adquirirlo? }\end{array}$ & Cantidad (fi) & $\begin{array}{c}\text { Porcentaje } \\
\text { (pi\%) }\end{array}$ \\
\hline $\begin{array}{l}\text { Por su } \\
\text { funcionalidad }\end{array}$ & 52 & $20 \%$ \\
\hline Por la marca & 69 & $27 \%$ \\
\hline $\begin{array}{l}\text { Por ser fácil de } \\
\text { conseguir }\end{array}$ & 40 & $15 \%$ \\
\hline Por su precio & 67 & $12 \%$ \\
\hline Por ser peruano & 259 & $100 \%$ \\
\hline Total & & \\
\hline
\end{tabular}

Por otro lado, en cuanto a los datos informativos que aparecen en el empaque de ambos productos (conservas de pescado y detergentes), la mayoría de las participantes (61\%) respondieron que sí leen esta información antes de adquirir dichos productos, mientras que el 39\% no consideran importante el leer dichos datos antes de comprar. Estos datos se aprecian en la Tabla 7.

Tabla 7 .

\section{Porcentajes de decisión} de compra de conservas

\begin{tabular}{|l|c|c|}
\hline $\begin{array}{l}\text { Pregunta 7: ¿Lee } \\
\text { datos informativos } \\
\text { del producto antes de } \\
\text { adquirirlos? }\end{array}$ & Cantidad (fi) & $\begin{array}{c}\text { Porcentaje } \\
\text { (pi\%) }\end{array}$ \\
\hline Sí & 158 & $61 \%$ \\
\hline No & 101 & $39 \%$ \\
\hline Total & 259 & $100 \%$ \\
\hline
\end{tabular}


Dentro de las participantes que leen los datos informativos antes de comprar los productos, las cuales fueron 158 mujeres; se obtuvo que el $34 \%$ solo leen la fecha de vencimiento y el $32 \%$ revisan el origen del producto, mientras que no conlleva mayor importancia los componentes o ingredientes (14\%).

\section{Tabla 8.}

Porcentajes de lectura de datos en el
empaque
\begin{tabular}{|l|c|c|}
\hline $\begin{array}{l}\text { Pregunta 8: } \\
\text { ¿Qué datos lee? }\end{array}$ & $\begin{array}{c}\text { Cantidad } \\
\text { (fi) }\end{array}$ & $\begin{array}{c}\text { Porcentaje } \\
\text { (pi } \%)\end{array}$ \\
\hline $\begin{array}{l}\text { Componentes o } \\
\text { ingredientes }\end{array}$ & 22 & $14 \%$ \\
\hline $\begin{array}{l}\text { Indicaciones o } \\
\text { formas de uso }\end{array}$ & 32 & $20 \%$ \\
\hline $\begin{array}{l}\text { Origen del } \\
\text { producto }\end{array}$ & 51 & $32 \%$ \\
\hline $\begin{array}{l}\text { Fecha de } \\
\text { vencimiento }\end{array}$ & 53 & $34 \%$ \\
\hline \multicolumn{1}{|c|}{ Total } & 158 & $100 \%$ \\
\hline
\end{tabular}

Por último, hemos analizado el segundo aspecto, el cual investiga el nivel de etnocentrismo y el conocimiento acerca del sello "Hecho en Perú", conformado entre las preguntas 9 y 16 del cuestionario. El nivel de etnocentrismo de las participantes se midió a través de una lista de afirmaciones las cuales tenían valores que fueron sumados para obtener el nivel de etnocentrismo, el cual podía encajar en 5 niveles dependiendo del valor final que alcanzase. En el caso de las mujeres del Cercado de Arequipa, más de la mitad de las encuestadas alcanzaron el segundo nivel: "Sí se puede compra productos nacionales" (53\%), mientras que ninguna $(0 \%)$ consiguió el nivel de "Importador Neto". Por consiguiente, podemos decir que la mayoría de las personas siempre que puedan compraran productos nacionales (ver Tabla 9).
Tabla 9.

\section{Porcentajes de nivel de etnocentrismo}

\begin{tabular}{|c|l|c|c|}
\hline $\begin{array}{c}\text { Pregunta } \\
\text { 9: Rango }\end{array}$ & $\begin{array}{c}\text { Nivel de } \\
\text { Etnocentrismo }\end{array}$ & $\begin{array}{c}\text { Cantidad } \\
\text { (fi) }\end{array}$ & $\begin{array}{c}\text { Porcentaje } \\
\text { (pi\%) }\end{array}$ \\
\hline$[10$ a 6] & Etnocentrista & 95 & $37 \%$ \\
\hline [5 a 1] & $\begin{array}{l}\text { Sí se puede } \\
\text { compra } \\
\text { productos } \\
\text { nacionales }\end{array}$ & 138 & $53 \%$ \\
\hline [0] & Indiferente & 15 & $6 \%$ \\
\hline -[1 a 5] & $\begin{array}{l}\text { Compra } \\
\text { cualquier } \\
\text { producto no } \\
\text { importa el } \\
\text { origen }\end{array}$ & 11 & $4 \%$ \\
\hline -[6 a 10] & $\begin{array}{l}\text { Importador } \\
\text { Neto }\end{array}$ & 0 & $0 \%$ \\
\hline & Total & 259 & $100 \%$ \\
\hline
\end{tabular}

En cuanto, al conocimiento acerca del sello "Hecho en Perú", se obtuvo que el 93\% de las participantes conocen que es el sello "Hecho en Perú", faltando solo 19 personas para ser el total de las participantes (ver tabla 10).

Tabla 10.

Porcentajes de conocimiento de sello "Hecho en Perú"

\begin{tabular}{|l|c|c|}
\hline $\begin{array}{l}\text { Pregunta 10: ¿Ha } \\
\text { escuchado hablar del } \\
\text { sello "Hecho en Perú"? }\end{array}$ & $\begin{array}{c}\text { Cantidad } \\
\text { (fi) }\end{array}$ & $\begin{array}{c}\text { Porcentaje } \\
\text { (pi\%) }\end{array}$ \\
\hline Sí & 240 & $93 \%$ \\
\hline No & 19 & $7 \%$ \\
\hline Total & 259 & $100 \%$ \\
\hline
\end{tabular}

Teniendo en cuenta solo a las 240 participantes que respondieron afirmativamente acerca de si conocían el sello "Hecho en Perú", el 85\% escuchó acerca de esta campaña por medio de medio televisivos; relegando a la radio (13.33\%), Internet (1.25\%) y por último, otros (o.42\%) como los afiches promocionales de distintos productos nacionales (ver Tabla 11). 
Tabla 11.

Porcentajes de medios de comunicación mediante se tiene conocimiento de sello "Hecho en Perú"

\begin{tabular}{|l|c|c|}
\hline $\begin{array}{l}\text { Pregunta 11: } \\
\text { ¿Qué medio de } \\
\text { comunicación } \\
\text { escucha la } \\
\text { propuesta "Hecho } \\
\text { en Perú"? }\end{array}$ & Cantidad (fi) & $\begin{array}{c}\text { Porcentaje } \\
\text { (pi\%) }\end{array}$ \\
\hline Televisión & 204 & $85.00 \%$ \\
\hline Radio & 32 & $13.33 \%$ \\
\hline Internet & 3 & $1.25 \%$ \\
\hline Otros & 1 & $0.42 \%$ \\
\hline Total & 240 & $100 \%$ \\
\hline
\end{tabular}

Además, para profundizar en su conocimiento acerca del tema se preguntó quién o quiénes promovían esta iniciativa aparte del sentimiento que los participantes pudiesen tener por la campaña. Por lo que, el $44 \%$ de las personas que decían conocer dicha campaña, respondieron que sí sabían quiénes son los promotores del sello "Hecho en Perú". Sin embargo, el $48 \%$ respondió negativamente, a pesar de no estar tan distanciados, podemos notar que más participantes no saben exactamente quienes promueven esta iniciativa.

\section{Tabla 12.}

Porcentajes de conocimiento de promotores del sello "Hecho en Perú"

\begin{tabular}{|l|c|c|}
\hline $\begin{array}{l}\text { Pregunta 12: ¿Conoce } \\
\text { quiénes son los } \\
\text { promotores de dicha } \\
\text { marca? }\end{array}$ & $\begin{array}{c}\text { Cantidad } \\
\text { (fi) }\end{array}$ & $\begin{array}{c}\text { Porcentaje } \\
\text { (pi\%) }\end{array}$ \\
\hline Sí & 115 & $44 \%$ \\
\hline No & 125 & $48 \%$ \\
\hline Total & 240 & $100 \%$ \\
\hline
\end{tabular}

Los participantes que respondieron positivamente a la pregunta anterior (115 mujeres), respondieron que los promotores de dicha marca son el Sector Privado (30\%) y el Estado Peruano (25\%), siendo estas las respuestas más utilizadas por las participantes de forma separada. Dejando en un porcentaje menor a los consumidores y las combinaciones de las dos primeras (ver Tabla 13).

Tabla 13.

Porcentajes de conocimiento de quiénes son los promotores del sello

"Hecho en Perú"

\begin{tabular}{|l|c|c|}
\hline $\begin{array}{l}\text { Pregunta 13: Los } \\
\text { promotores de la } \\
\text { marca Perú son }\end{array}$ & $\begin{array}{c}\text { Cantidad } \\
\text { (fi) }\end{array}$ & $\begin{array}{c}\text { Porcentaje } \\
\text { (pi\%) }\end{array}$ \\
\hline a) El Estado Peruano & 29 & $25 \%$ \\
\hline b) Sector Privado & 34 & $30 \%$ \\
\hline c) Consumidores & 18 & $16 \%$ \\
\hline d) a y b & 19 & $17 \%$ \\
\hline e) a y c & 15 & $13 \%$ \\
\hline Total & 115 & $100 \%$ \\
\hline
\end{tabular}

En cuanto, al sentimiento que les produce dicha marca, el 36\% de las participantes respondieron que sienten orgullo por lo peruano y un $35 \%$ piensan que es importante para el comercio nacional la promoción de los productos locales. Además, se obtuvo que el $18 \%$, piensa que esta marca es buena para impulsar el emprendimiento de los peruanos en tema de negocios, para que los peruanos tengan mayor oportunidad de entrar al mercado con productos nacionales. A pesar, de que hubo un $11 \%$ de mujeres que respondieron sentirse indiferentes ante esta iniciativa, resulta siendo el menor porcentaje de la muestra (ver Tabla 14). 
Tabla 14.

Porcentajes de sentimiento que produce el sello "Hecho en Perú"

\begin{tabular}{|l|c|c|}
\hline $\begin{array}{l}\text { Pregunta 14: ¿Cuál es } \\
\text { el sentimiento que le } \\
\text { produce el sello "Hecho } \\
\text { en Perú"? }\end{array}$ & $\begin{array}{c}\text { Cantidad } \\
\text { (fi) }\end{array}$ & $\begin{array}{c}\text { Porcentaje } \\
\text { (pi\%) }\end{array}$ \\
\hline Orgullo Peruano & 86 & $36 \%$ \\
\hline $\begin{array}{l}\text { Es importante para } \\
\text { el comercio nacional }\end{array}$ & 84 & $35 \%$ \\
\hline $\begin{array}{l}\text { Impulsa el } \\
\text { emprendimiento } \\
\text { peruano }\end{array}$ & 44 & $18 \%$ \\
\hline Indiferente & 26 & $11 \%$ \\
\hline Total & 240 & $100 \%$ \\
\hline
\end{tabular}

Por otra parte, las participantes creen que el propósito del sello "Hecho en Perú" es la promoción y publicidad (35\%) y su identificación para diferenciarlos de los productos extranjeros. Solo el 9\% creen que es para mejorar la competitividad de los productos peruanos en el mercado (ver Tabla 15).

\section{Tabla 15.}

Porcentajes de conocimiento de propósito del sello "Hecho en Perú"

\begin{tabular}{|l|c|c|}
\hline $\begin{array}{l}\text { Pregunta 15: ¿Cuál } \\
\text { cree usted que es el } \\
\text { propósito principal } \\
\text { del Sello "Hecho en } \\
\text { Perú? }\end{array}$ & $\begin{array}{c}\text { Cantidad } \\
\text { (fi) }\end{array}$ & $\begin{array}{c}\text { Porcentaje } \\
\text { (pi\%) }\end{array}$ \\
\hline $\begin{array}{l}\text { Identificar a productos } \\
\text { peruano }\end{array}$ & 80 & $33 \%$ \\
\hline $\begin{array}{l}\text { Satisfacer a los clientes } \\
\text { peruanos }\end{array}$ & 56 & $23 \%$ \\
\hline $\begin{array}{l}\text { Promoción y } \\
\text { publicidad }\end{array}$ & 83 & $35 \%$ \\
\hline $\begin{array}{l}\text { Mejorar la } \\
\text { competitividad de los } \\
\text { productos peruanos }\end{array}$ & 21 & $9 \%$ \\
\hline Total & 240 & $100 \%$ \\
\hline
\end{tabular}

Por último, a las participantes se les preguntó ¿cuánto les gustaba el sello "Hecho en Perú”?, a lo que, el $40 \%$ respondió que les gusta la iniciativa y el $33 \%$ que le encantaba, por lo que podemos concluir que al $73 \%$ de las participantes ven a esta campaña de forma positiva. Mientras que solo el $17 \%$ y $10 \%$ manifestó que lo detestaba o simplemente no le gustaba, sumando el $27 \%$ del total de la muestra (ver Tabla 16).

Tabla 16.

Porcentajes de gusto por el sello "Hecho en Perú"

\begin{tabular}{|l|c|c|}
\hline $\begin{array}{l}\text { Pregunta 16: } \\
\text { ¿Cuánto le gusta } \\
\text { el Sello "Hecho en } \\
\text { Perú"? }\end{array}$ & Cantidad (fi) & $\begin{array}{c}\text { Porcentaje } \\
\text { (pi\%) }\end{array}$ \\
\hline Lo detesto & 41 & $17 \%$ \\
\hline No me gusta & 25 & $10 \%$ \\
\hline Me gusta & 96 & $40 \%$ \\
\hline Me encanta & 78 & $33 \%$ \\
\hline Total & 240 & $100 \%$ \\
\hline
\end{tabular}

\section{Discusión}

De acuerdo con los resultados obtenidos mediante esta investigación, analizando la variable de la influencia del sello "Hecho en Perú” en la decisión de compra de los productos de conservas de pescado y detergentes podemos concluir que el 100\% de los participantes de la investigación conocía las marcas mostradas de dichos productos teniendo conocimiento de la variedad para su elección a la hora de comprar. Además, obtuvimos que la marca preferida fue "Campomar" (28\%) en cuanto a conservas de pescado, atribuyendo que su elección para la compra de este producto es con $24 \%$ la nacionalidad del producto y sus variadas presentaciones. De este resultado podemos concluir que en el caso de conservas de pescado la mayoría elige 
“Campomar" teniendo en cuenta que es un producto nacional aunque no resulta un factor decisivo en el momento de la compra. Por otro lado en el caso de los detergentes la marca preferida fue "Ariel" (28\%) seguido de cerca por "Sapolio" (26\%), atribuyendo dicha preferencia a la marca reconocida $(27 \%)$ y a la nacionalidad del producto (26\%). Así en este producto podemos observar que es muy importante la marca en la decisión de compra en el caso de "Ariel"; pero al mismo tiempo el origen del producto es un factor importante en el caso de "Sapolio".

En cuanto a los datos informativos que aparecen en el empaque de ambos productos (conservas de pescado y detergentes), la mayoría de las participantes (61\%) respondieron que leen la información de la etiqueta a la hora de adquirir dichos productos. El dato más consultado es la fecha de vencimiento (34\%), seguido de cerca por el origen del producto (32\%).

Analizando los resultados en cuanto a la variable de etnocentrismo en el consumo, podemos decir que el $53 \%$ de las mujeres participantes en el estudio obtuvieron resultados entre el rango de 1 a 5, el cual viene a ser "sí se puede compran productos nacionales", por lo que podemos concluir que el nivel de etnocentrismo en el Cercado de Arequipa es moderado y se encuentra en un puesto importante dentro de la mente del consumidor antes de adquirir un producto. Comparando dicho resultado con otras investigaciones de otros países, tal como Chile (Schnettler et al., 2008; Schnettler et al., 2010), Colombia (Concha et al., 2011) y España (Guerrero et al., 2010), que tienen un tema de estudio similar al de la presente investigación; observamos que en Chile también es un factor importante el origen del producto antes de adquirirlo y que tienen un etnocentrismo al consumo moderado ya que tienen siempre presente el valor de comprar productos nacionales. En cambio, en Colombia y España, los factores más importantes a la hora de comprar son las características del producto, beneficios que ofrece al consumidor y además de su calidad y frescura; toma en cuenta todos los factores antes mencionados antes que el origen del producto. Sin embargo es preciso detallar que nuestra investigación se realizó bajo estándares generales sobre la percepción del etnocentrismo al consumo, mientras que las investigaciones anteriormente mencionadas basaban su percepción de acuerdo al tipo de producto ofrecido. Lo cual nos lleva a presumir que el etnocentrismo en dichas investigaciones se vio afectado por el tipo de producto investigado.

Analizando la variable del conocimiento acerca del sello "Hecho en Perú", se obtuvo que el 93\% de las participantes conocen el sello "Hecho en Perú", y que lo conocen a través de medios televisivos (85\%). Pero, el $48 \%$ desconocen quienes son los promotores de la iniciativa, por lo que podemos decir que sólo conocen la marca en nombre y no profundizan su conocimiento del tema. Además nos demuestra que la campaña de promoción de la marca no llegó a toda las población peruana. Entre aquellas personas que respondieron afirmativamente, la mayoría piensa que el "Sector Privado" es el promotor de la campaña (30\%), sin embargo, esta respuesta estaría incompleta, ya que los promotores son el "Estado" y el "Sector Privado" (17\%), por lo cual, deducimos que a pesar de su conocimiento sobre el tema, no tienen la idea completa 
acerca de todo lo que representa dicha campaña. Además podemos inferir que muchas de las participantes marcaron afirmativamente sin tener el conocimiento necesario. Otro punto importante también referido a la campaña es que la mayoría de personas pensaban que el sello "Hecho en Perú" es para promoción y publicidad con un $35 \%$, mientras que un $33 \%$ señaló que su propósito era identificar los productos nacionales, y únicamente un $9 \%$ coincidieron con el objetivo de la campaña, que era aumentar la competitividad de los productos nacionales.

En cuanto al aspecto subjetivo referente al sello, el 36\% de las participantes respondieron que sienten orgullo por lo peruano y el $35 \%$ creen que es importante para el comercio nacional de los productos. Además que el sello demuestra tener gran acogida ya que más de un 70\% de la población afirmó que le gustaba (40\%) o le encantaba (33\%). Esto nos indica que si bien la población no conoce el propósito del sello es de mucho agrado para la población por lo cual los promotores de la campaña pueden utilizar este aspecto a su favor para difundir aún más cuál es el verdadero propósito del sello "Hecho en Perú".

\section{Referencias}

Agbonifoh, B. \& Elimimian, J. U. (1999). Attitudes of developing countries toward "country of origin" products in an era of multiple brands. "Journal of International Consumer Marketing”, 11(4), 97-116.

El Comercio. (2009). "La nueva era de 'Cómprale al Perú', la campaña para el consumo de lo nacional”. (Documento en formato html). El Comercio, 29 de julio. Recuperado el 31 de octubre del 2012 de: http://elcomercio.pe/economia/319535/noticia-nueva-era-comprale-al-peru-campana-consumo-lo-nacional

El Peruano (2011). “Cómprale al Perú”. El Peruano, 29 de julio. (Documento en formato html) Recuperado el $31 \mathrm{de}$ octubre del 2012: www.elperuano.com. pe/edicion/noticia.aspx?key=ezCMpmd $4 \mathrm{WiU}=$

Ferrell, O. C. \& Hartline, M. D. (2006). "Estrategía de marketing”. Madrid: Cengage Learning Editores.

Gestiopolis. (2010). "Características del producto". (Documento en formato html) Recuperado el 7 de octubre del 2012 de: www.gestiopolis.com/marketing/caracteristicas-del-producto.htm

Guerrero, J. F.; Abad, J. C. \& Agüera, E. D. (2010). La importancia del "país de origen” en la fase de evaluación del consumidor en productos hortícolas. "Revista Española de estudios Agrosociales y Pesqueros”, 225, 47-69.

Gurhan-Canli, Z. \& Maheswaran, D. (200o). Cultural variations in country of origin effects. "Journal of Marketing Research”, 37, 309-317.

INDECOPI. (2009). "Cómprale al Perú". (Documento en formato pdf). Recuperado el 1 de noviembre del 2012: http://www.indecopi.gob.pe/repositorioaps/o/14/par/guiasinfor/hecho_ peru.pdf) 
Jiménez, A. I. \& Calderón, H. (2004). “Dirección de productos y marcas". Barcelona: UOC.

Lampert, S. \& Jaffe, E. (1998). A dynamic approach to country of origin effect. "European Journal of Marketing", 32(1), 61-78.

López, F. (2008). "Proceso de decisión del consumidor". Madrid: ESIC.

Ministerio de la Producción. (2006). "Cómprale al Perú". (Documento en formato html). Recuperado el 28 de octubre del 2012: http://www.compralealperu.gob.pe/quienes.php

Montesinos, M., \& Curras, R. (s/f). Importancia relativa de la marca y efecto país de origen sobre la intención de compra de los consumidores. "Decisiones de marketing" (Documento en formato pdf). Recuperado el 29 de octubre del 2012 de: dialnet.unirioja.es/descarga/ articulo/2499417.pdf

Nagashima, A. (1970). A comparison of Japanese and U.S. attitudes toward foreign products. "Journal of Marketing", 34, 68-74.

Schnettler, B.; Ruiz, D. \& Sepúlveda, O. (2008). La importancia del país de origen en el consumo de alimentos. "Panorama Socioeconómico", 23, 50-56.

Schnettler, B.; Cid, E.; Miranda, H. \& Sepúlveda, J. D. (2010). Etnocentrismo y efecto país de origen en la compra de arroz en supermercados de las ciuda- des de Los Ángeles y Temuco, Chile. Panorama Socioeconómico, 28(40), 18-32.

Shimp, T. \& Sharma, S. (1987). Consumer ethnocentrism: Construction and validation of the CETSCALE. "Journal of Marketing Research", 3(24), 280-289.

Schooler, R. D. (1965). Product bias in Central American Common Market. "Journal of Marketing Research", 2(4), 394-397.

Sosa, J. C. y Ortiz, M. (s/f). "El efecto del país de origen en la intención de compra: un modelo multidimensional". (Documento en formato pdf). Recuperado el 26 de octubre del 2012 de: http://quest.uprrp.edu/7quest/ papers/Juan\%2oCarlos\%2oSosa\%20 Varela.pdf

Supphellen, M. y Gronhaug K. (2003) Building foreign brand personalities in Russia: the moderating effect of consumer ethnocentrism "International Journal of Advertising", 22, 203-226.

Supphellen, M. y Rittenburg T. (2001). Consumer ethnocentrism when foreign products are better. "Psychology \& Marketing”, 12, 907-927.

Usunier, J. C. (2006). Relevance in business research: The case of country-of-origin research in marketing. "European Management Review", 3, 60-73.

Concha, J. R.; Gómez, E. \& Valencia, P. $(\mathrm{s} / \mathrm{f})$. El efecto país de origen entre los consumidores colombianos: El 
caso de un producto de consumo masivo. "I Simposio de Investigación ASCOLFA". (Documento en formato pdf) Recuperado el 1 de diciembre del 2012 de: http://www.ascolfa. edu.co/archivos/2.6\%20M\%20-\%20 CONCHA,\%20GOMEZ\%20\%20y\%20 VALENCIA\%20(P).pdf

Recibido: 08-09-2015

Aceptado: 31-10-2015 